\title{
An Exploration of Nucleic Acid Liquid Biopsy Using Glucose Meter
}

\author{
Wei Zhao, Yu Gu, Cong-Hui Xu, Jing-Juan Xu and Hong-Yuan Chen \\ State Key Laboratory of Analytical Chemistry for Life Science and Collaborative Innovation Center of \\ Chemistry for Life Sciences, School of Chemistry and Chemical \\ Engineering, Nanjing University, Nanjing 210023, China. \\ E-mail: weizhao@nju.edu.cn (W. Zhao), xujj@nju.edu.cn (J.J. Xu).
}

\begin{abstract}
:
The development of non-invasive techniques for the diagnosis of cancer, characterization of mutation and monitoring treatment response could greatly reduce the morbidity and mortality caused by cancer. Nevertheless, the extremely low amount of cell free nucleic acids makes the liquid biopsy a very challenging task. Herein, taking advantage of the pocked size, reliable quantitative results and simple operation of the pocket-sized personal glucose meter (PGM), we report an approach of circulating microRNA-21 (miR-21) detection with high precision and low cost. Via target-induced release of invertase from the DNA-invertase conjugate, which could convert sucrose into glucose, detection of miR-21 in serum was linked to PGM reading. Combined DNAzyme feedback amplification (DFA) program and highly efficient enzymatic turnover, an ultralow detection limit of $7 \times 10^{-16} \mathrm{M}$ for miR-21 was achieved using PGM as the reporter. The high sensitivity and selectivity of the proposed method meets the requirement of quantifying cell free nucleic acids in serum. In addition, this approach fills the shortage of quantitative RT-PCR and next-generation sequencing in quantifying miRNAs with short length, and greatly reduces the cost of detection. We believe the widely used personal diagnosis devices could hold an important place in the booming area of liquid biopsy.
\end{abstract}

Key words: liquid biopsy, microRNA-21, DNAzyme feedback amplification, personal glucose meter, nucleic acids

Liquid biopsy, an innovative technology in cancer testing, is a non-invasive approach, which could relieve the suffering of patients from conventional biopsy, decrease false positive rate because of the tissue sample bias and help oncologists gain a broader molecular understanding of the disease. ${ }^{[1,2]}$ Nucleic acids, including cell free DNA (cfDNA), circulating mRNA as well as microRNA (miRNA) are the promising biomarkers in blood for cancer screening, monitoring drug treatment response, and postoperative assessment. ${ }^{[3]}$ However, due to the relative rarity of circulating nucleic acids against the huge background in plasma or serum samples, liquid biopsy still faces challenges including the extraction and enrichment of target biomarkers, and increasing the sensitivity of detection methods.

Here we show a proof-of-concept strategy for circulating miRNA assay with personal glucose meter (PGM). As a widely used personal diagnosis device for point-of-care testing (POCT), PGM benefits from its pocket size, reliable performance and simple operation. The main challenge is the relative low sensitivity of PGM for the analysis of circulating nucleic acid. To achieve an ultra-high sensitivity, amplification strategy of DNAzyme feedback amplification (DFA) was applied, which took advantage of rolling-circle amplification (RCA) and an RNA-cleaving DNAzyme (RCD). Unlike RT-PCR, the whole RCA program could be initiate by a short nucleic acid and generate hundreds of tandemly linked copies of a covalently closed circle in a few minutes under isothermal conditions. The produced RCD elements from RCA triggered the production of more input complexes for RCA. The autonomous reaction circuit resulted in exponential DNA amplification. As suggested 
by Brennan and $\mathrm{Li}$, sensitivity improvements of 3-6 orders of magnitude over conventional methods could be achieved for miRNA sensing by DFA. ${ }^{[4]}$ Further, the DFA product was hybridized with DNA-invertase conjugates, and efficiently catalyze the hydrolysis of sucrose into glucose, which is quantified by a PGM. The proposed method provided ultralow detection limit of $7 \times 10^{-16} \mathrm{M}$ miRNA-21 and large dynamic range over 4 orders. Added miRNA-21 in blood serum was determined using the proposed method with high selectivity. The PGM coupled with DFA program holds promising potential in ultra-sensitive and low-cost liquid biopsy.

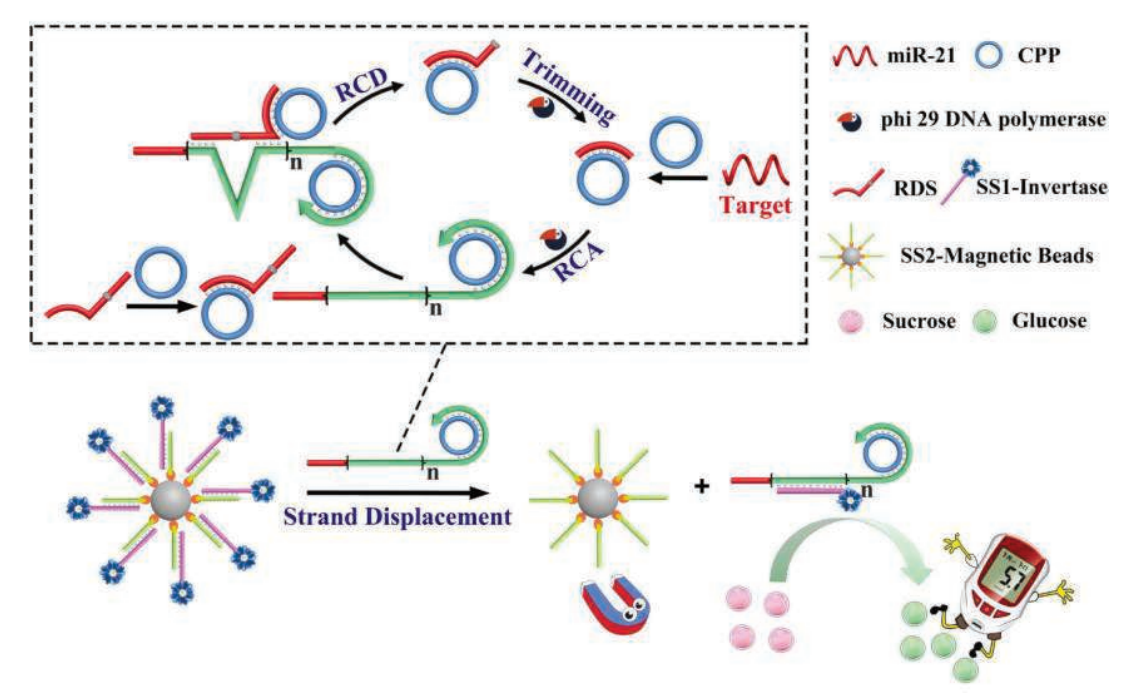

Fig.1. Principle of miR-21 sensing based on DFA and target-induced release of invertase.

\section{References}

[1] H. Schwarzenbach, D. S. B. Hoon and K. Pantel, Nature Reviews Cancer, 11, 426-437 (2011); doi:10.1038/nrc3066

[2] B. L. Khoo, G. Grenci, Y. B. Lim, S. C. Lee, J. Han and C. T. Lim, Nature Protocols, 13, 34-58 (2018); doi:10.1038/nprot.2017.125

[3] J. Kaiser, Science 327, 1074-1074 (2010); doi: 10.1126/science.327.5969.1074

[4] M. Liu, Q. Zhang, D. Chang, J. Gu, J. D. Brennan and Y. Li, Angewandte Chemie International Edition, 56, 6142-6146 (2017); doi: 10.1002/anie.201700054 